\title{
Renormalization Group for Disordered Dirac Fermions
}

\author{
André LeClair* \\ Newman Laboratory, Cornell University, Ithaca, NY 14850. \\ E-mail: 'Leciair@mail.ins. corneì. edù
}

ABSTRACT: We use the recently proposed all orders beta functions to study localization problems in two dimensions. A classification of random Dirac hamiltonians is presented. Beta functions for the Chalker-Coddington network model are presented and it is described how the renormalization group flows are toward a true singularity.

The main subject of this talk is localization-delocalization transitions in two dimensions. This is a subject with many challenging and unsolved problems, the most important being finding a theoretical description of the critical properties of the quantum Hall transition. The Chalker-Coddington network model is perhaps the simplest model believed to be in the universality class of the quantum Hall transition [i] of random Dirac fermions, and it is believed that the disorder averaged effective theory should possess an infra-red fixed point conformal field theory capable of predicting the

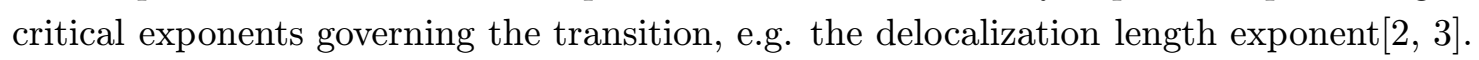

I will describe an attempt at finding the expected fixed point based on a proposed all

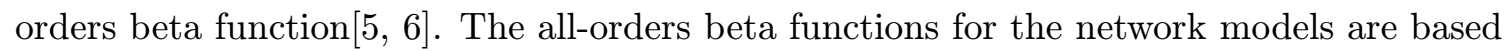

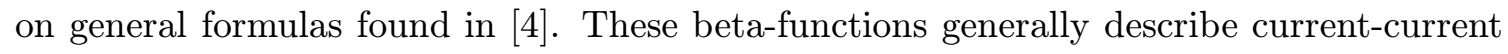
interactions in two dimensions. Though they remain conjectural, several strong checks of the formula were performed for the simplest $s u(2)$ case in $\left[\overline{i_{1}}\right]$. As I will describe, for reasons that are not understood at the time, but appear to be specific to the application of the general theory to disordered systems, the renormalizaton group flows toward a true singularity, and thus we did not find the expected fixed point.

The plan of the talk is as follows. I will first describe a classification of Random Dirac Fermions in 2d. In the next section I explain how supersymmetric Effective Actions are obtained upon disorder averaging. These generally involve current-current interactions and in the next section I describe the renormalization group (RG) for general interactions of this kind. Using arguments independent of the detailed beta functions, I then propose a connection between the possible fixed points and the Virasoro Master equation. In the last sections these methods are applied to the network models for the quantum Hall transtions. Unfortuanately, as I will describe, the all orders beta functions that appear to be correct for $s u(2)$ predict flows toward true singularities in the disordered problems.

${ }^{*}$ Speaker. 


\section{Classification of Random Dirac Hamiltons}

Most interesting localization problems in $2 d$ can be formulated as spectral problems for Dirac-like Hamiltonians. Some well-known examples are the following:

-Quantum Hall Transition. The simplest model in this universality class is the ChalkerCoddington network model [iij.

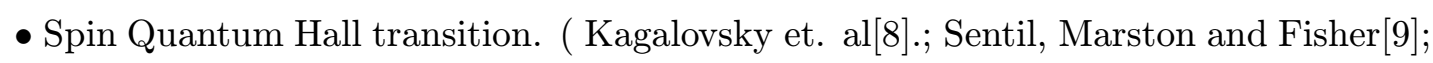

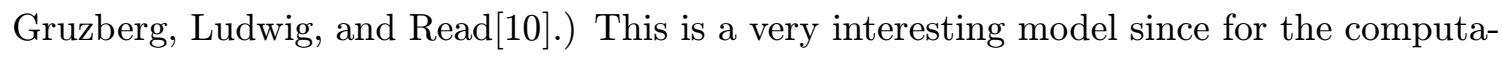
tion of some quantities the model can be mapped onto percolation [i $\left[\overline{1} \overline{0}^{i}\right]$. It can thus serve as a important test case when developing more general continuum methods.

-Quasi-particle localization in systems with degenerate Fermi surfaces and dirty su-

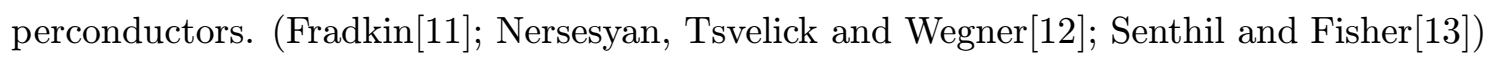

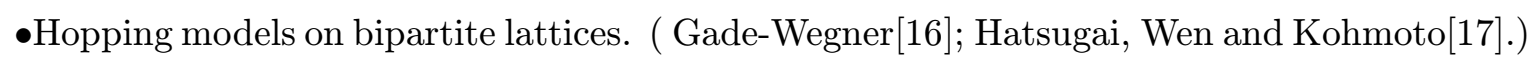

Altland and Zirnbauer gave a classification of random matrices (Hamiltonians) based on the discrete symmetries of time-reversal, particle-hole symmetry and chirality. A basic question is the following: is the classification of universality classes of random Dirac Hamiltonians equivalent to the Altland-Zirnbauer classification of random matrices? We actually found 13 classes instead of Altland and Zirnbauer's 10. Let me describe our classification, which was published in [i] $\left[\begin{array}{l}\overline{8} \\ \mathbf{8}\end{array}\right]$.

Discrete symmetries and the Classification. A hamiltonian with Dirac structure can always be written as

$$
H=\left(\begin{array}{cc}
V_{+}+V_{-} & -i \partial_{\bar{z}}+A_{\bar{z}} \\
-i \partial_{z}+A_{z} & V_{+}-V_{-}
\end{array}\right)
$$

where $A_{z}, A_{\bar{z}}, V_{ \pm}$are random matrices. The classification is based on the following discrete symmetries:

-Chirality: $\quad H=-P H P^{-1}, \quad P^{2}=1$

-Particle-hole: $\quad H=-C H^{T} C^{-1}, \quad C^{T}= \pm C$

•Time-reversal: $\quad H=K H^{*} K^{-1}, \quad K^{T}= \pm K$

The result of analyzing the various possibilities is shown in the following table. 


\begin{tabular}{|c|c|c|c|c|c|}
\hline & $\begin{array}{c}\text { Random matrix } \\
\text { class }\end{array}$ & $\begin{array}{c}\text { Time rev. } \\
\text { inv. }\end{array}$ & $\mid \begin{array}{l}\text { Part.-hole } \\
\text { symmetry }\end{array}$ & Chirality & $\begin{array}{l}\text { Sym. } \\
\text { group }\end{array}$ \\
\hline class 0 & $\mathbf{A}=\mathrm{GUE}$ & no & no & no & $U(n)$ \\
\hline class 1 & $\mathbf{A}_{\mathbf{I I I}}=$ chiral GUE & no & no & yes & $U(n)$ \\
\hline class 2 & $\mathbf{A}_{\mathbf{I I I}}=$ chiral GUE & no & no & yes & $U(n) \times U(n)$ \\
\hline class $\mathbf{3}_{+}$ & $\mathbf{A}_{\mathbf{I I}}=\mathrm{GSE}$ & yes & no & no & $O(n)$ \\
\hline class $3_{-}$ & D & no & yes & no & $O(n)$ \\
\hline class $\mathbf{4}_{+}$ & $\mathbf{A}_{\mathbf{I}}=\mathrm{GOE}$ & yes & no & no & $S p(2 n)$ \\
\hline class $\mathbf{4}_{-}$ & $\mathrm{C}$ & no & yes & no & $S p(2 n)$ \\
\hline class 5 & $\mathbf{D}_{\mathbf{I I I}}=$ chiral GOE & yes & yes & yes & $O(n)$ \\
\hline class 6 & $\mathbf{C}_{\mathbf{I}}=$ chiral GSE & yes & yes & yes & $S p(2 n)$ \\
\hline class 7 & $\mathbf{D}_{\mathbf{I I I}}=$ chiral GOE & yes & yes & yes & $O(n) \times O(n)$ \\
\hline class 8 & $\mathbf{C}_{\mathbf{I}}=$ chiral GSE & yes & yes & yes & $S p(2 n) \times S p(2 n)$ \\
\hline class $\mathbf{9}_{+}$ & $\mathrm{D}_{\mathrm{I}}$ & yes & yes & yes & $U(n)$ \\
\hline class $\mathbf{9}_{-}$ & $\mathrm{C}_{\text {II }}$ & yes & yes & yes & $U(n)$ \\
\hline
\end{tabular}


As an example of the distinction between the classification of random matrices, Classes 1, 2 are both chiral GUE as far as the Altland-Zirnbauer classification. But here:

Class 1: $\quad A \in g l(n), \quad V_{ \pm}=0$.

Class 2: $\quad A=\operatorname{diag}\left(a_{+}, a_{-}\right), \quad a_{ \pm} \in g l(n)$.

$$
V_{ \pm}=\left(\begin{array}{cc}
0 & w_{ \pm} \\
w_{ \pm}^{\dagger} & 0
\end{array}\right), \quad w_{ \pm} \in \operatorname{gl}(n)
$$

The chiral classes 1, 5, 6 are pure gauge, i.e. the only non-zero potential is $A \in$ $g l(n), s o(n)$, or $s p(2 n)$. Classes $0,3_{+}, 4_{+}$are the Wigner-Dyson classes GUE, GOE, GSE.

Classification of non-Hermitian Dirac Hamiltonians. Here we can also impose

$$
H= \pm Q H^{\dagger} Q^{-1}, \quad Q^{2}=1
$$

In this case we found 87 classes with Dirac structure and 43 classes of random matrices [i] $[\overline{1} \overline{9}]$ ]

\section{Disorder averaged effective action $S_{e f f}$}

Using the supersymmetric method to perform the disorder averaging, we need to consider the action:

$$
S=i \int \frac{d^{2} x}{2 \pi}\left(\psi^{\dagger} H \psi+\beta^{\dagger} H \beta\right)
$$

$\psi$ are fermionic fields and $\beta$ bosonic ghost fields. $S_{\text {eff }}$ is obtained from gaussian functional integrals over the random potentials $A, V_{ \pm}$and depends on the couplings $g_{1}, g_{2}, \ldots$ which are variances of the disordered potentials.

The resulting effective action can always be expressed in the following form:

$$
S_{e f f}=S_{c f t}+\int \frac{d^{2} x}{2 \pi} \sum_{A} g_{A} d_{a b}^{A} J^{a} \bar{J}^{b}
$$

Here, $S_{c f t}$ is an $\operatorname{osp}(2 n \mid 2 n)_{1}$ supercurrent algebra conformal field theory with currents $J^{a}(z), \bar{J}^{a}(\bar{z})$, where $n$ is the number of fermions. ( $k=1$ is the level).

The above method can be applied to all classes listed in the previous section. The important examples we will focus on are the following:

-Chalker-Coddington network model: Class 0 with $n=1$. This is a 3-coupling perturbation of $\operatorname{osp}(2 \mid 2)_{1}$.

-Spin Quantum Hall transition: Class $4_{-}$(class C) with $n=2$. This is a 3 -coupling perturbation of $\operatorname{osp}(4 \mid 4)_{1}$. 


\section{All-orders $\beta$ eta function for general current-current perturbations}

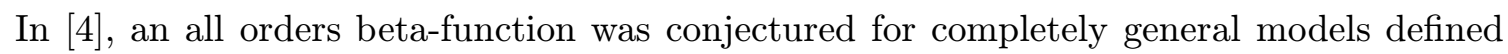
by the action:

$$
S=S_{G_{k}}+\int \frac{d^{2} x}{2 \pi} \sum_{A} g_{A} \mathcal{O}^{A}
$$

Above, $G_{k}$ is a level $k$ current algebra for the (super) group $G$, with currents $J^{a}$, and the perturbing operators are left-right current-current interactions:

$$
\mathcal{O}^{A} \equiv d_{a b}^{A} J^{a} \bar{J}^{b}
$$

The $\beta$ eta function depends on some structure constants $C, D, \widetilde{C}$ which are easily computed in the cft. To compute these structure constants it is convenient to also define the purely chiral operator:

$$
T^{A} \equiv d_{a b}^{A} J^{a} J^{b}
$$

The following operator product expansions are valid in the conformal theory:

$$
\begin{gathered}
\mathcal{O}^{A}(z, \bar{z}) \mathcal{O}^{B}(0) \sim \frac{1}{z \bar{z}} C_{C}^{A B} \mathcal{O}^{C}(0) \\
T^{A}(z) \mathcal{O}^{B}(0) \sim \frac{1}{z^{2}}\left(2 k D_{C}^{A B}+\widetilde{C}_{C}^{A B}\right) \mathcal{O}^{C}(0)
\end{gathered}
$$

The conformal Ward identities for the currents allow one to isolate the log divergences that contribute to the beta function in an efficient manner. To two loops one finds:

$$
\beta_{g_{A}}=-\frac{1}{2} g_{B} g_{C} C_{A}^{B C}-\frac{k}{2} g_{B} g_{C} g_{D} D_{E}^{B C} \widetilde{C}_{A}^{E D}+\ldots
$$

The one-loop result is well-known, but the two loop result is new. Gerganov, Moriconi and I proposed the all-orders formula:

$$
\beta_{g}=-\frac{1}{2} C\left(g^{\prime}, g^{\prime}\right)\left(1+k^{2} D^{2} / 4\right)+\frac{k^{3}}{8} C\left(g^{\prime} D, g^{\prime} D\right) D-\frac{k}{2} \widetilde{C}\left(g^{\prime} D, g\right)
$$

The notation is as follows: $\bullet g=$ a row vector

$\bullet D=$ matrix, $\quad D_{B}^{A}=\sum_{C} D_{B}^{A C} g_{C}$

- $C(a, b)=$ a row vector, $C(a, b)_{A}=\sum_{B, C} a_{B} b_{C} C_{A}^{B C}$

$\bullet g^{\prime}=g /\left(1-k^{2} D^{2} / 4\right)$

The simplest example is anisotropic $s u(2)$, which corresponds to the well known Kosterlitz-Thouless flows:

$$
\sum_{A} g_{A} \mathcal{O}^{A}=g_{1}\left(J^{+} \bar{J}^{-}+J^{-} \bar{J}^{+}\right)+g_{2} J_{3} \bar{J}_{3}
$$


The result is:

$$
\begin{aligned}
\beta_{g_{1}} & =\frac{g_{1}\left(g_{2}-g_{1}^{2} / 4\right)}{\left(1-g_{1}^{2} / 16\right)\left(1+g_{2} / 4\right)} \\
\beta_{g_{2}} & =\frac{g_{1}^{2}\left(1-g_{2} / 4\right)^{2}}{\left(1-g_{1}^{2} / 16\right)^{2}}
\end{aligned}
$$

\section{Resolving the RG flows[i].}

Though the $\beta$ eta function has pole singularities, the flows are not singular. Flows that approach the poles do so along non-singular trajectories and pass smoothly through the pole. All RG flows can be extended to arbitrarily large and small length scales using:

-Strong-weak coupling duality. Let $g^{*} \equiv 16 / g$ then the $\beta$ eta function satisfies:

$$
\beta^{*}\left(g^{*}\right)=\frac{\partial g^{*}}{\partial g} \beta_{g}=-\beta\left(g \rightarrow g^{*}\right)
$$

The poles occur at the self-dual points $g=g^{*}=4$. If $g=g^{*}$ at some scale $r_{0}$, then $g$ flows to $g^{*}$ at scale $r_{0} / r$.

-Topological identification We need to identify $g_{2}= \pm \infty$. One way of seeing this is that the dual to these flows are completely smooth and continuous.

-RG invariant Remarkably the flows possess the invariant:

$$
Q=\frac{g_{1}^{2}-g_{2}^{2}}{\left(g_{2}-4\right)^{2}\left(g_{1}^{2}-16\right)}
$$

All the flows are shown in the following figure. There are regions that are known to correspond to massive and massless sine-Gordon theories and massive sinh-Gordon theory.

A few remarks on the status of the conjectured beta-function are in order. A number of important checks were performed in $[\bar{i}] \overline{1}]$. The most sensitive check was of the massless flows that arise in the imaginary sine-Gordon theory defined by $g_{2} \rightarrow i g_{2}$. The above beta-function correctly predicts the known non-perturbative relation between the anomalous dimensions in the UV and IR. An all orders beta-function was also proposed by Al. Zamolodchikov (unpublished) and his result was quoted in [2020]. Zamolodchikov's argument was global in nature and did not rely on summing up perturbation theory; the main input was the known properties of the massless flows, thus his beta-function appears to be the unique one up to a change of coordinates that captures the non-perturbative aspects of the flows. It can be shown that the beta-function in $\left[2 \overline{2}{ }^{i}\right]$ is equivalent to the above under a change of coordinates. In summary, though the beta-function remains a conjecture, these tests provide some confidence in applying them to the network models. 


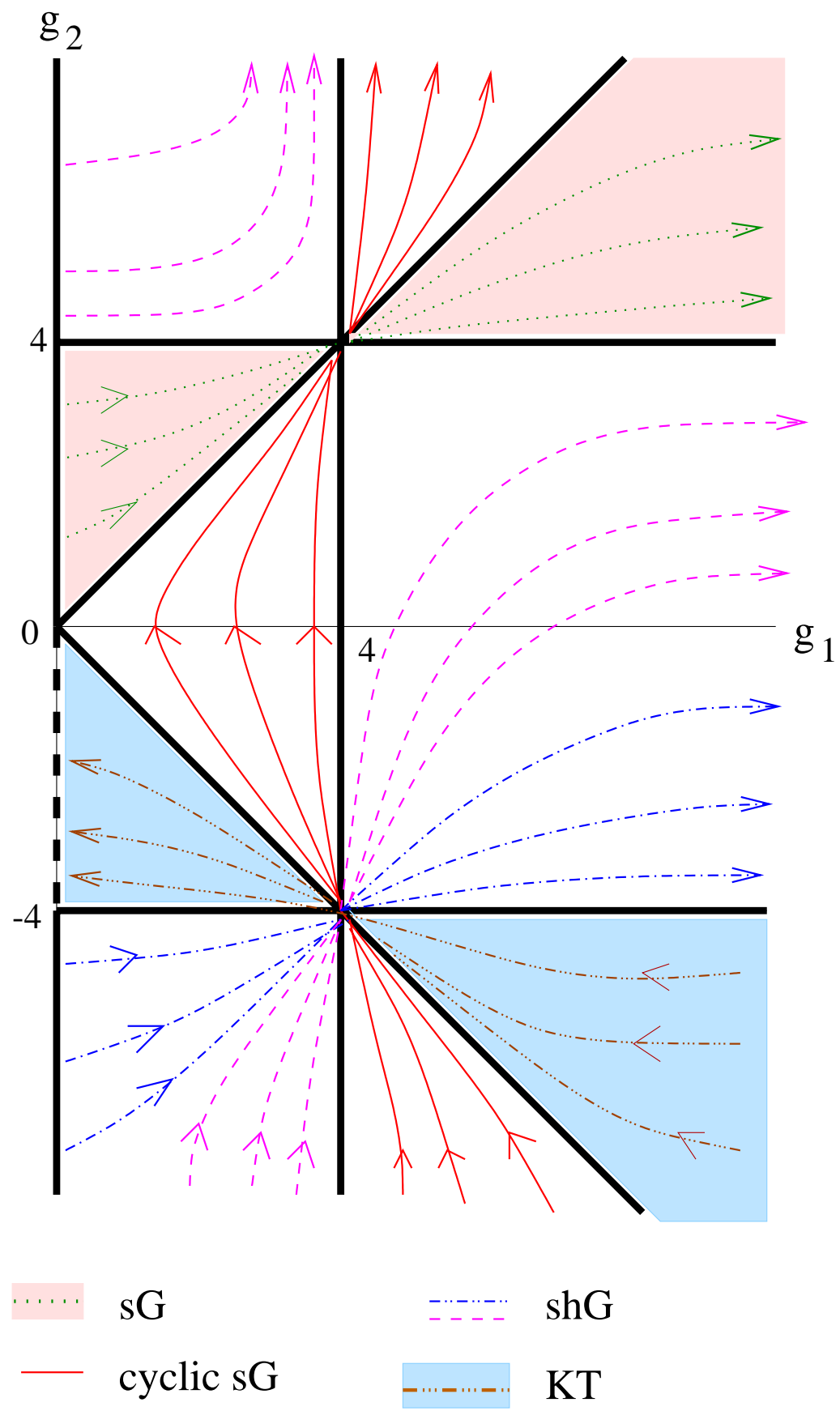

\section{Fixed points and the Virasoro Master Equation (VME)}

Before studying the beta functions for the network models, we can ask what kinds of fixed points can we expect for general current-current perturbations? A way to study this that does not rely on the $\beta$ eta function is based on the VME. Classically the theories have a traceless stress tensor:

$$
T_{\text {classical }}=T_{G_{k}}-g_{A} T^{A}
$$


where $T_{G_{k}}$ is the affine Sugawara stress tensor. Let us make the hypothesis that the fixed points correspond to

$$
T_{\text {fixed point }}=\sum_{A} h_{A} T^{A}
$$

where the $h_{A}$ are constants. In order for $T$ to satisfy the Virasoro algebra one finds

$$
\left(2 k D_{C}^{A B}+C_{C}^{A B}+2 \widetilde{C}_{C}^{A B}\right) h_{A} h_{B}=h_{C}
$$

Note that these are the same structure constants that appear in the beta function. The above equation is the VME of Halpern et. al.[2] $\left.\overline{1}_{-1}\right]$. •Generic solutions to the VME correspond to current algebra cosets. There exists a physically appealing RG mechanism for realizing such a coset fixed point: Under the RG flow the couplings may be attracted to a submanifold of couplings corresponding to a subgroup $H$ of $G$. This coupling can then flow to infinity, thereby gapping out $H$. The fixed point would then be the coset:

$$
\text { fixed point }=\frac{G_{k}}{H_{k^{\prime}}}
$$

\section{RG flows for the network models}

For the Chalker-Coddington network model the $\beta$ eta functions are

$$
\begin{aligned}
& \beta_{g_{+}}=\frac{8 g_{+}\left(g_{+}^{2}\left(2 g_{a}-g_{-}+2\right)+2 g_{-}\left(2-g_{-}\right)+8 g_{a}\right)}{\left(4-g_{+}^{2}\right)\left(2-g_{-}\right)^{2}} \\
& \beta_{g_{-}}=\frac{8 g_{+}^{2}\left(2+g_{-}\right)^{2}}{\left(4-g_{+}^{2}\right)^{2}} \\
& \beta_{g_{a}}=\frac{4\left(\left(g_{+}^{2}-g_{-}^{2}\right)\left(16-g_{+}^{2} g_{-}^{2}\right)+4 g_{a} g_{+}^{2}\left(2+g_{-}\right)\left(2-g_{-}\right)^{2}\right)}{\left(4-g_{+}^{2}\right)^{2}\left(2-g_{-}\right)^{2}}
\end{aligned}
$$

where $g_{ \pm}$are variances of $V_{ \pm}$and $g_{a}$ is the variance of the $u(1)$ gauge field $A$. Though these are complicated beta functions, we analyzed the flows in detail, and found that they possess the following properties $\left[\overline{\hat{\sigma}_{i}}\right]$ :

-Unlike the $s u(2)$ case, there are some truely singular flows in the physical regime.

- There are also some regular flows that can be extended to all scales using duality and topological identifications.

$$
\begin{gathered}
\text { duality : } \quad g_{ \pm}^{*}=\frac{4}{g_{ \pm}}, \quad g_{a}^{*}=-\frac{4 g_{a}}{g_{-}^{2}} \\
\text { self - dual points : } \quad\left(g_{+}, g_{-}, g_{a}\right)=( \pm 2, \pm 2,0)
\end{gathered}
$$

- The singular flows are attracted to $g_{ \pm}=2, g_{a} \neq 0$ Since $g_{a} \neq 0$ is not self dual, the flows cannot be extended beyond the singularity using duality. 
-Some flows attracted to $g l(1 \mid 1) \in \operatorname{osp}(2 \mid 2)$ submanifold. This is a realization of the $\operatorname{osp}(2 \mid 2)_{1} / g l(1 \mid 1)_{1}$ solution of VME. This phase is essentially the disordered XY model[[2 $\left.2 \overline{2}\right]$.

These flows are depicted in the following 3 figures.
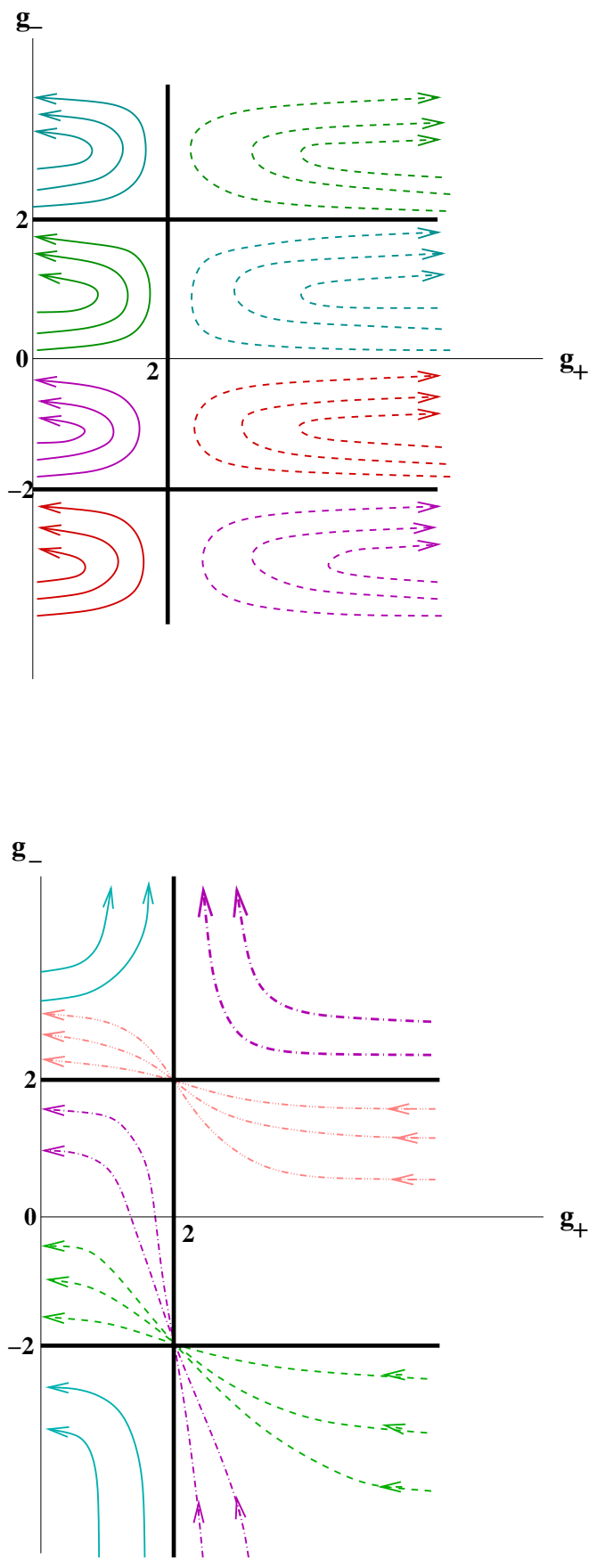


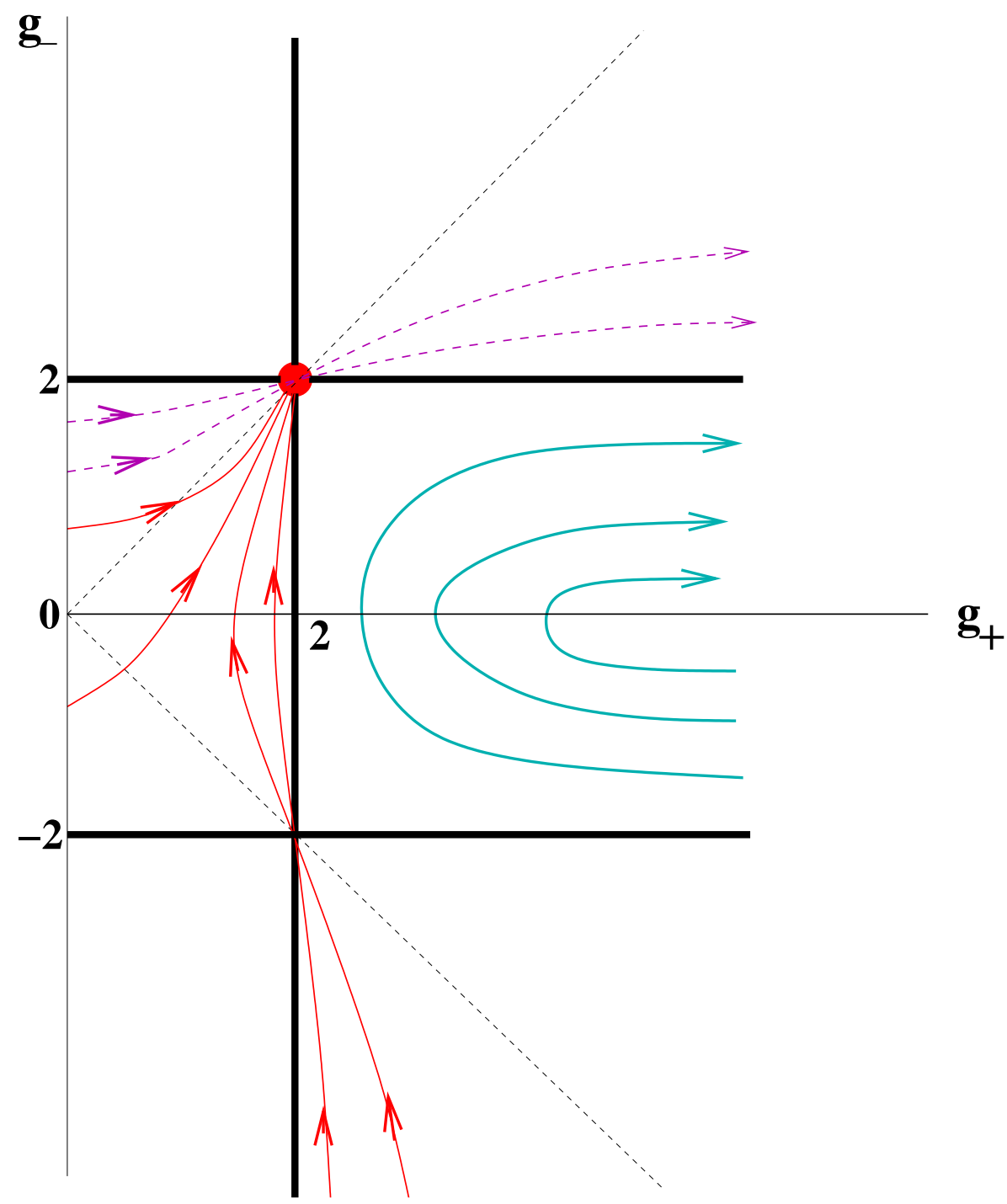

It turns out that the beta functions for the network model of the spin Quantum Hall transition have very similar properties. We will defer a discussion of the possible meaning of the singular flows to the conclusions.

\section{VME for Network Models}

As we saw in the last section, the beta functions do not lead to the identification of any 
fixed points in the physical regimes of the network models. Let us turn then to the solutions of the VME. For the spin quantum Hall effect (SQHE) there exists the solution:

$$
S Q H E: \quad \frac{o s p(4 \mid 4)_{1}}{s u(2)_{0}}
$$

As explained in [i $[\overline{6}]$ this leads to a density of states exponent:

$$
\overline{\rho(E)} \sim E^{1 / 7}
$$

Encouragingly, this agrees with the map to percolation [i [i $\left.0_{1}^{1}\right]$. The above coset can be shown to correspond precisely to the current algebra $\operatorname{osp}(2 \mid 2)_{-2}$ at level $-2[\overline{2} \overline{2} \overline{3}]$. However recent arguments were given that the true fixed point cannot be a current algebra[ [2 24$]$.

For the usual QHE, the analog of the above coset is:

$$
Q H E: \quad \frac{o s p(2 \mid 2)_{1}}{u(1)_{0}}
$$

This leads to a density of states exponent:

$$
\Longrightarrow \overline{\rho(E)}=\text { constant }
$$

which is known to be correct for the QHE.

\section{Multifractal exponents from Cosets}

Another promising feature of the above cosets is that they lead to multi-fractality in a very natural way. Consider $N$ copies of the theory. It turns out the beta functions are the same, i.e. independent of $N$ because of supersymmetry. The $N$-copy solutions of the VME are the following cosets:

$$
\begin{array}{cl}
\text { QHE }: & \frac{o s p(2 N \mid 2 N)_{1}}{u(1)_{0}} \\
\text { SQHE : } & \frac{o s p(4 N \mid 4 N)_{1}}{s u(2)_{0}}
\end{array}
$$

To study wavefunction multi-fractality, one considers the moments:

$$
P^{(q)}=\frac{\int d^{2} x \overline{\langle\rho\rangle^{q}}}{\left(\int d^{2} x \overline{\langle\rho\rangle}\right)^{q}}
$$

The latter can be computed in the $N$ copy theory for any $N \geq q$ and should be independent of $N$. This is a non-trivial property and is certainly not automatic. It turns out that the cosets have this property! From the above moments, one conventionally defines the exponent $\alpha_{0}$ as follows:

$$
P_{t y p}=\exp (\overline{\log \rho}) \sim L^{-\alpha_{0}}
$$


As explained in [23i], for the SQHE, the above coset leads to $\alpha_{0}=2+1 / 4$. Recent numerical work gave the value $\alpha_{0} \approx 2+1 / 8[25 i]$. Unfortunately, this seems to rule out the coset as the proper fixed point.

One may also consider conductance multifractality by studying higher moments of the two-point functions:

$$
\begin{gathered}
G^{(q)}=\overline{\langle\rho(r) \rho(0)\rangle^{q}} \\
G_{t y p}=\exp (\overline{\log G}) \sim r^{-X_{t}}
\end{gathered}
$$

It can be shown that in any theory with a constant density of states [i2 $\left.\overline{2}_{1}^{1}, \overline{2} \overline{2} \overline{3}\right]$ the exponents $X_{t}$ and $\alpha_{0}$ are related as follows:

$$
X_{t}=2\left(\alpha_{0}-2\right)
$$

The above relation agrees well with numerical simulations in the QHE which give $X_{t} \approx 1 / 2$, $\alpha_{0} \approx 9 / 4[\overline{2} \overline{2} \overline{6}]$. An rather weak argument was given in [6] $[\overline{6}]$ for the value $\alpha_{0}=9 / 4$ for the QHE based on the possibility of considering the twist fields that modify boundary conditions. However since the coset for the SQHE appears not to be in the right universality class, it is unlikely that this argument is correct.

\section{Discussion}

In summary, the all orders beta functions proposed in [i, [i] $]$ work very nicely for $s u(2)$ but predict a flow towards a true singularity after a finite RG time for the network models of Quantum Hall transitions, and thus failed to identify the expected fixed point. Since it was expected that this theory should possess the correct fixed point, this failure needs to be understood, and one can hope that the proper resolution of the difficulties we encountered will point further investigations in the right direction. It is likely that the singularity of the flows can be traced to the level zero current algebras that are present in the supersymmetric approach to disorder averaging. Another possibility is that the singularities are related to the freezing transitions that are known to occur in even the pure random gauge field

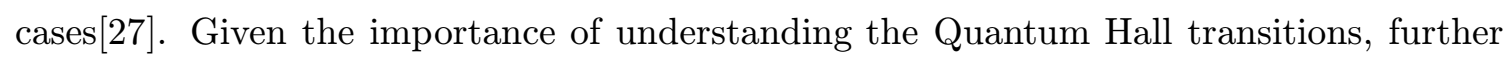
investigation into the nature of the singular flows is called for.

\section{References}

[1] J. T. Chalker and P. D. Coddington, J. Phys. C 21 (1988) 2665.

[2] A. W. W. Ludwig, M. P. A. Fisher, R. Shankar and G. Grinstein, Phys. Rev. B50 (1994) 7526.

[3] C.-M. Cho and J. T. Chalker, Phys. Rev. B 54, 8708 (1996).

[4] B. Gerganov, A. LeClair and M. Moriconi, Phys.Rev.Lett. 86 (2001) 4753.

[5] A. LeClair, Phys.Rev. B64 (2001) 045329.

[6] D. Bernard and A. LeClair, Nucl.Phys. B628 (2002) 442-472. 
[7] D. Bernard and A. LeClair, Phys.Lett. B512 (2001) 78-84.

[8] V. Kagalovksy, B. Horovitz, Y. Avishai and J. T. Chalker, Phys. Rev. Lett 82 (1999) 3516.

[9] T. Senthil, J. B. Marston and M. P. A. Fisher, Phys. Rev. B 60 (1999) 4245.

[10] I. A. Gruzberg, A. W. W. Ludwig and N. Read, Phys. Rev. Lett. 82 (1999) 4524.

[11] E. Fradkin, Phys. Rev. B33 (1986) 3257; Phys. Rev. B33 (1986) 3263;

[12] A.A. Nersesyan, A.M. Tsvelik and F. Wegner, Phys. Rev. Lett. 72 (1994) 2628;

[13] T. Senthil and M.P.A. Fisher, Phys. Rev. B 60 (1999) 6893, arXiv:cond-mat/9810238;

[14] T. Senthil and M.P.A. Fisher, Phys. Rev. B 61 (1999) 6893; M. Bocquet, D. Serban and M.R. Zirnbauer, Nucl. Phys. B 578 (2000) 628;

[15] A. Altland, B.D. Simons, M.R. Zirnbauer, cond-mat/0006362;

[16] R. Gade and F. Wegner, Nucl. Phys. B 360 (1991) 213; R. Gade, Nucl. Phys. B398 (1993) 499.

[17] Y. Hutsugai, X.-G. Wen, M. Kohmoto, Phys. Rev. B56 (1997) 1061.

[18] D. Bernard and A. LeClair, cond-mat/0109552.

[19] D. Bernard and A. LeClair, cond-mat/0110649.

[20] S. Lukyanov, "Low energy effective Hamiltonian for the XXZ spin chain", cond-mat/9712314.

[21] M. B. Halpern, E. Kiritsis, N. A. Obers and K. Clubok, Phys. Rep. 265, 1 (1996).

[22] S. Guruswamy, A. LeClair and A. W. W. Ludwig, Nucl. Phys. B 583, 475 (2000).

[23] D. Bernard and A. LeClair, cond-mat/0003075, Phys.Rev. B64 (2001) 045306

[24] N. Read and H. Saleur, Nucl.Phys. B613 (2001) 409, hep-th/0106124.

[25] A. D. Mirlin, F. Evers and A. Mildenberger, cond-mat/0208451.

[26] F. Evers, A. Mildenberger and A. D. Mirlin, cond-mat/0105297.

[27] D. Carpentier and P. Le Doussal, Phys.Rev. E63 (2001) 026110. 